\title{
Coming to grips with weight perception: Effects of grasp configuration on perceived heaviness
}

\author{
J. RANDALL FLANAGAN and CHRISTINA A. BANDOMIR \\ Queen's University, Kingston, Ontario, Canada
}

\begin{abstract}
We investigated how changes in grasp configuration affect perceived heaviness in a weight discrimination task in which participants compared the weights of a series of test objects with the weight of a reference object. In different experiments, we varied the width of the grasp, the number of digits employed, the angle of the grasp surface, and the size of the contact area between the digits and the object. We show that objects are perceived to be lighter when lifting with (1) a wide grip in comparison with a narrow grip, (2) five digits in comparison with two digits, and (3) a large contact area in comparison with a small contact area. However, the angle of the contact surfaces did not influence perceived weight. We suggest that changes in central motor commands associated with grasp differences may influence perceived weight, at least under some conditions.
\end{abstract}

The human hand is well adapted for both perceiving and manipulating the world within our grasp. The numerous skeletal and muscular degrees of freedom of the hand, orchestrated by highly developed neural control systems, provide for tremendous dexterity that allows for both delicate exploration and versatile manipulation of objects (Lemon, 1993). The hand is also richly endowed with a variety of sensory receptors, including cutaneous mechanoreceptors, that provide precise information about forces applied by the hand, slips between the object and skin, and hand position (e.g., Edin \& Johansson, 1995; Johansson \& Westling, 1987). This sensory information enables the hand to appreciate object properties, such as weight, compliance, and slipperiness, and is also critical in object manipulation (see Johansson, 1996, and Johansson \& Cole, 1994, for reviews), as is illustrated by the fact that individuals with sensory deficits have great difficulty handling small objects (e.g., Moberg, 1962; Rothwell et al., 1982).

The tight coupling between action and perception by the hand has long been recognized. Weber's (1834/1996) early demonstration that weight discrimination is more accurate when actively lifting an object than when passively supporting it established that the use of muscular information contributes to weight perception, a finding since confirmed by many studies (see Jones, 1986, for a review). Although there is ongoing debate about the relative contributions to weight perception of efferent (central) and afferent (peripheral) signals associated with muscular effort (Lansing \& Banzett, 1993; Matthews,

This study was supported by the Canadian Natural Sciences and Engineering Research Council (NSERC). Then authors thank David Murray and Helen Ross for their helpful comments. Correspondence should be addressed to J. R. Flanagan, Department of Psychology, Queen's University, Kingston, ON, K7L 3N6, Canada (e-mail: flanagan@psyc. queensu.ca).
1982), the evidence suggests that both signals can influence perceived weight or force under appropriate conditions (Brodie \& Ross, 1984; Jones, 1986).

Most studies of weight perception assume that it is the muscular exertion directly involved in lifting that contributes to weight perception. However, Kilbreath and Gandevia (1991) have shown that the perceived heaviness of a reference weight, lifted by one digit, increases if a concurrent weight (equal to or greater than the reference) is lifted by any other digit of the same hand. In contrast, Charpentier (1891; Murray, Ellis, Bandomir, \& Ross, 1999 ) reported that a weight lifted with one hand alone feels heavier than when another weight is lifted, at the same time, by the other hand or some other part of the body. In a previous study (Flanagan, Wing, Allison, \& Spencely, 1995), we provided further evidence that muscles only indirectly involved in lifting can influence weight perception. We asked participants to compare the weights of objects, covered in either slippery satin or less slippery sandpaper, by lifting using a precision grip with the tips of the thumb and index finger on either side (see Figure 1A). When lifting with this grasp, grip forces, normal to the contact surfaces and in the horizontal plane, must be applied to generate friction between the skin and the contact surface and prevent the object from slipping. Johansson and Westling (1984) have shown that, in precision lifting, grip force is precisely scaled for both the weight of the object and the friction between the digit and the object. Grip force is also precisely adjusted for changes in inertial load during movement (Flanagan \& Wing, 1993, 1995; see Wing, 1996, for a review). We have shown that objects covered in satin are judged to be heavier than objects of equal weight covered in sandpaper (Flanagan et al., 1995). We suggested that the increase in perceived weight is caused by a failure to distinguish between the effort involved in lifting the object and the effort involved in applying grip forces to stabilize the object in hand. That is, 


\section{Grasps and Objects}
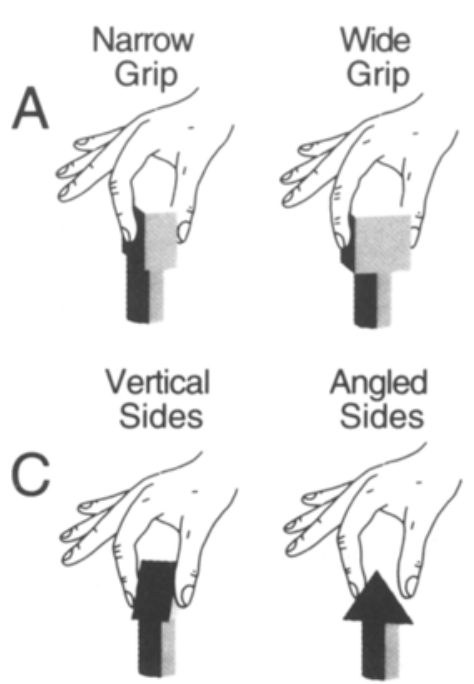

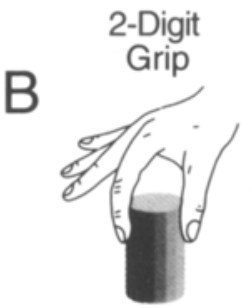

Large Contact Area





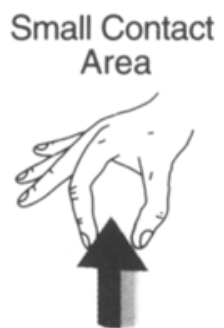

Figure 1. Schematic diagram showing the four pairs of grip configurations used in this study and the objects lifted in the weight discrimination task. We varied the width of the grip (A), the number of digits employed $(B)$, the angle of the contact surface $(C)$, and the size of the contact area between the digits and the object (D).

we argued that the additional grip force required to lift the more slippery object led to an increase in perceived weight. Rinkenauer, Mattes, and Ulrich (1999) have recently replicated our finding that increases in grip force due to surface friction are associated with increases in perceived weight. By examining a range of surface frictions, these authors were able to demonstrate that the strength of this "surface-weight" illusion (as they referred to it) does not depend on a simple linear sum of grip force and weight but instead depends on a weighted sum of the logarithms of grip force and weight.

In the present study, we examined how grasp configuration affects the perceived weight of an object. Our general hypothesis is that an object will be perceived to be heavier when the total effort involved in lifting increases. We define effort as the level of central or efferent motor drive (Jones, 1986) associated with lifting. By total effort we mean the central drive associated with both lifting forces and forces only indirectly involved in lifting (e.g., grip force). Our hypothesis assumes that perceived weight depends, at least in part, on central signals related to lifting. Specifically, it is assumed that a copy of the efferent signal (efference copy) generates a sensation (corollary discharge) that influences perceived weight. We refer to this centrally generated sensation as the sense of effort. We manipulated the total effort involved in lifting by varying grasp configuration. Specifically, we varied grip width, the number of digits employed, the angle of the grasp surfaces, and the size of the contact area between the digits and object (see Figure 1). These changes in grasp configuration were designed to alter either the magnitude of the forces indirectly involved in lifting or the efficiency with which these forces could be generated (i.e., the effort required to generate a given force). Both of the factors influence the total effort associated with lifting.

In the first two experiments, we examined the effects of grip width on perceived weight. We hypothesized that an object would be judged to be lighter when grasped with a wide grip than when grasped with a narrow grip (see Figure 1A). This hypothesis is based on recent results suggesting that a wide grip is more efficient (i.e., requires less effort) than a narrow grip. Recently, Van Doren (personal communication, September 1998) has shown that the amount of electromyographic (EMG) activity required to sustain a given grip force depends on the span between the thumb and opposing fingers. In particular, he found that, for a given grip force, EMG activity in the first dorsal interosseus (1DI) increased monotonically as the grasp span decreased from $8 \mathrm{~cm}$ to about $0.5 \mathrm{~cm}$. Assuming that EMG activity provides an estimate of the level of central motor drive, this result suggests that less effort (central motor drive) will be required to grasp an object with a wide grip than with a narrow grip. Consistent with this suggestion, Van Doren (1998) has also shown that if effort is held constant, more grip force can be generated with a wider grip. Van Doren asked subjects to grasp a test object and then delivered perturbations that widened or narrowed the grip. In order to keep effort (central drive) constant before and after the perturbation, subjects were instructed "not to intervene" following the perturbation (i.e., do not make a voluntary correction). Van Doren reported that grip force increases when the grip is widened and decreases when the grip is narrowed. He concluded that, for the same effort, greater grip force can be generated with a wider grip.

In the third experiment, we examined whether perceived weight depends on the number of digits used to 
grasp the object. Kinoshita, Murase, and Bandou (1996) reported that when holding a cylindrical object from above (as shown in Figure 1B), the total normal force decreases with the number of digits employed. The same finding has been reported when holding a rectangular object with the thumb in opposition with one to four digits (Kinoshita, Kawai, \& Ikuta, 1996). The decrease in total normal force is likely the result of an increase in grasp stability as digits are added. On the basis of these previous results, we hypothesized that an object would be judged heavier when lifted with two digits than when lifted with five digits because the total normal force, and, hence, the total effort required to perform the task, would be greater.

Jenmalm and Johansson (1997) have recently shown that the fingertip forces used to lift an object are greater when its grasp surfaces are angled than when they are vertical (see Figure 1C). In particular, the horizontal forces required to prevent the object from slipping are substantially greater when the contact surfaces are angled than when they are vertical. Therefore, the total effort required to lift by the angled surfaces will be greater. Our general hypothesis that weight perception depends on total effort predicts that an object will be perceived to be heavier when grasped by its angled surfaces than when grasped by its vertical surfaces. We tested this prediction in the fourth experiment. In this experiment, we observed that some subjects appeared to use a larger contact area (i.e., the area between the digits and the object) when grasping the angled surfaces. Therefore, in the final experiment, we examined the effects of contact area on perceived weight (see Figure 1D). The potential influence of contact area on weight perception was noted by Weber (1834/1996), and, later, Charpentier (1891; Murray et al., 1999) observed that when an object is placed successively on a series of flat lightweight surfaces contacting the hand, the perceived weight of the object decreases with the size of the surface. This suggests that perceived weight may vary inversely with contact area. However, in our experiment, we varied the size of the digit contact surfaces, and it is unclear whether a surface-weight illusion will be observed under these conditions.

Of the five experiments reported in this paper, three (Experiments 1, 3, and 4) were planned in advance, whereas two (Experiments 2 and 5) were carried out to further investigate issues raised in Experiments 1 and 4, respectively. Therefore, we report results based on two different groups of participants. However, we stress that the same method of recruitment was used for the two groups of participants, and they were treated in much the same way (see below).

\section{EXPERIMENT 1 Effect of Grip Width on Perceived Weight}

In Experiment 1, we tested the hypothesis that an object would be judged to be heavier when grasped with a narrow grip than when grasped with a wide grip because the total effort involved would be greater. This hypothesis is based on recent observations that greater effort (cen- tral drive) is required to generate grip force when a narrow grip is employed (Van Doren, 1998, and personal communication, September 1998). It is important, however, to consider other mechanisms whereby grip width may contribute to weight perception. One such mechanism is the size-weight illusion. When lifting large and small objects of equal weight, people judge the small object to be heavier than the large object (Charpentier, 1891; Murray et al., 1999) even when they are aware that the two objects are equal in weight (Flournoy, 1894). Ellis and Lederman (1993) have demonstrated that vision is not necessary for the size-weight illusion and that haptic information about object size - obtained from grasping the objects - is both necessary and sufficient to obtain the full strength of the illusion. To guard against a contribution (to perceived weight) of the size-weight illusion, we varied grip width while holding the size of the object constant. Participants were asked to compare the weights of rectangular blocks (all of the same size) while grasping either the wide or the narrow side with a wide or narrow grip, respectively (Figure 1A). The participants viewed the objects while lifting and could appreciate that they were all equal in size. Therefore, any effects of grip width on perceived weight ought not to be caused by the size-weight illusion.

\section{Method}

Participants. Forty undergraduates ( 26 women and 14 men) between the ages of 18 and 25 years participated in this experiment after giving informed consent. All participants were recruited from the Psychology 100 subject pool at Queen's University and received credit toward their final grade for their participation. None of the participants reported sensory or muscular problems, and all had normal or corrected-to-normal vision. The participants in Experiment 1 also completed Experiments 3 and 4, and the order of experiments was counterbalanced across participants.

Stimuli. The objects consisted of a set of rectangular wooden blocks mounted on top of 35-mm-film canisters (Figure 1A). The blocks were $7 \mathrm{~cm}$ long, $3 \mathrm{~cm}$ wide, and $5 \mathrm{~cm}$ high. The film canisters were filled with small tire weights mixed into Playdough. The Playdough prevented the tire weights from moving when objects were lifted. The Playdough plus tire weights were thoroughly mixed such that the mass was approximately evenly distributed throughout the canisters. There were nine test objects ranging in weight from 96 to $176 \mathrm{~g}$. The increment between the seven central test objects was $8 \mathrm{~g}$, and the increment between the two lightest and two heaviest test objects was $16 \mathrm{~g}$. Thus, the nine weights were 96,112 , $120,128,136,144,152,160$, and $176 \mathrm{~g}$. There was also one reference object that weighed $136 \mathrm{~g}$, the central value of the test objects.

Procedure. Each participant completed four sets of nine trials. In each set, the participant was asked to compare the weights of the nine test objects with the weight of the reference object. In each trial, the experimenter placed a randomly selected test object and the reference object on a table covered in felt to muffle any sounds that could provide cues to weight. The participants were asked to use their preferred hand to lift the reference object followed by the test object and then indicate whether the test object was lighter or heavier than the reference. (The participants were not allowed to indicate that objects were the same weight.) The participants were encouraged to make their decision after a single lift of each object but were allowed extra lifts. They were encouraged to lift the object "straight up" and not to tilt the object. Lifting was accomplished by whole arm motion (the forearm was not supported) and primarily involved rotation about the shoulder. No specific instructions were 
given regarding whether to move the object after lifting. However, the majority of participants simply lifted the object, held it for a moment in a stationary position, and then replaced it on the tabletop.

The objects were grasped using a precision grip with the tips of the thumb and index finger on either side (Figure 1A). The participants were encouraged to grasp the center of the object (as shown in the figure). In the first two sets of trials, the participants lifted both the test and the reference objects with either the narrow ("narrownarrow" condition) or the wide ("wide-wide" condition) grip. These trials will be referred to as same trials because the same grip width was used to lift the test and reference objects. In the second two sets of trials, the participants were asked to lift the reference object with a narrow grip and lift the test objects with a wide grip ("narrowwide" condition), or vice versa ("wide-narrow" condition). These trials will be called different trials. The order of the sets of same and different trials was counterbalanced across participants.

Analysis. For each of the four conditions (narrow-narrow, widewide, narrow-wide, wide-narrow), the probability of responding heavier, $p \mathrm{H}$, was determined for each test object (i.e., weight). The probability is simply the ratio of the number of participants who responded heavier divided by the total number of participants. The $p \mathrm{H}$ was then plotted as a function of the weight of the test object.

Logit analysis (McCullagh \& Nelder, 1989) was used to test for differences in the $p \mathrm{H}$ functions between grip widths. Separate comparisons were carried out for the same and different trials. The following logit model was used to estimate $p \mathrm{H}$ as a function of object weight and grip width used for the test objects:

$$
p \mathrm{H}=\frac{\exp \left(\beta_{0}+\beta_{1} G+\beta_{2} \text { Weight }\right)}{1+\exp \left(\beta_{0}+\beta_{1} G+\beta_{2} \text { Weight }\right)},
$$

where the dummy variable $G$ codes for grip width ( $G=-1$ for narrow, and $G=1$ for wide). The above equation can be transformed into the following linear model:

$$
\log \left(\frac{p \mathrm{H}}{1-p \mathrm{H}}\right)=\beta_{0}+\beta_{1} \mathrm{G}+\beta_{2} \text { Weight }
$$

By evaluating the estimated value of $\beta_{1}$, it is possible to test whether the $p \mathrm{H}$ functions obtained for the two grip widths are reliably different. Note that the above model assumes that the two $\mathrm{pH}$ curves are parallel (i.e., equally steep) but may be shifted with respect to one another. Therefore, tests for parallelism were also carried out to investigate possible interactions between grip width and weight. As will be shown below, the additive linear model shown in Equation 2 provided a good fit to the data from all five experiments.

\section{Results and Discussion}

The results of Experiment 1 are shown in Figure 2. Figure $2 \mathrm{~A}$ shows $p \mathrm{H}$ values and estimated functions obtained when the reference and test objects were both lifted with either a narrow (open circles and thin trace) or a wide (filled circles and thick trace) grip. As expected, when the test object was $96 \mathrm{~g}$, the probability of responding that the test object is heavier than the reference $(p \mathrm{H})$ was close to 0 for both grips widths, and when the test object was $176 \mathrm{~g}$, the probability was 1 . Note that when the test object was the same weight as the reference $(136 \mathrm{~g}), p \mathrm{H}$ was greater than .5 . The tendency to judge the second of two equally weighted objects to be heavier is known as the time error and dates back to Fechner (see review by Hellström, 1985). We also observed the time error in our previous studies on weight discrimination using similar prócedures (Flanagan \& Wing, 1997; Flanagan et al., 1995).
Regression analysis revealed that the coefficient for grasp type ( $\beta_{1}$ in Equation 2$)$ was not reliably different from zero $[t(17)=1.16, p=.26]$. Thus, there was not a reliable difference in the horizontal location of the two $p \mathrm{H}$ functions shown in Figure 2A. Moreover, the test for parallelism did not reveal a reliable interaction between grasp type and weight $\left[\chi^{2}(1)=1.91, p=.17\right]$. This indicates that the $p \mathrm{H}$ functions obtained for the narrownarrow and wide-wide conditions were equally sensitive to changes in weight.

Figure 2B shows the $p \mathrm{H}$ values and estimated curves obtained when the reference and test objects were lifted either with a wide and a narrow grip (open circles and thin line) or with a narrow and a wide grip (filled circles and thick line), respectively. Overall, for a given test weight, $p \mathrm{H}$ tended to be greater when the test object was lifted with a narrow grip than with a wide grip. Regression analysis confirmed that the $p \mathrm{H}$ curve for the narrow-wide condition was significantly shifted to the right of the $p \mathrm{H}$ curve for the wide-narrow condition $[t(17)=-2.25$, $p=.038]$. The test for parallelism applied to these different trials failed to reveal a reliable interaction between weight and grip width $\left[\chi^{2}(1)=0.46, p=.50\right]$. The horizontal shift between the two $p \mathrm{H}$ functions corresponds to a difference in perceived weight of the test objects of $3.85 \mathrm{~g}$. (For any given value of $p \mathrm{H}$, the difference between the two functions is given by the ratio of $\beta_{1}$ to $\beta_{2}$.) This indicates that, on average, an object grasped with the wide grip must be $3.85 \mathrm{~g}$ heavier than an object grasped with a narrow grip to be perceived to be the same weight. By comparison, the difference in perceived weight between the two same condition $p \mathrm{H}$ functions (wide-wide vs. narrow-narrow) was $-0.11 \mathrm{~g}$ (not significant). Note that any effect of grip width on perceived weight should result in larger differences in $p \mathrm{H}$ when the test and reference weights are similar. As shown in Figure $2 \mathrm{~B}$, the largest difference in $p \mathrm{H}$ occurred when the test object was equal in weight to the reference object.

The results obtained in this experiment indicate that when an object is grasped with a wide $(7-\mathrm{cm})$ grip, it appears lighter than when grasped with a narrow $(3-\mathrm{cm})$ grip. We suggest that this may be due to fact that the wider grip is more efficient in terms of generating normal forces required to prevent the object from slipping. As noted above, Van Doren (1998) has reported that the muscle activity required to sustain a given grip force increases as the span between the thumb and opposing fingers decreases from $8 \mathrm{~cm}$ to about $0.5 \mathrm{~cm}-\mathrm{a}$ range that includes the grip widths we examined. This suggests that the central motor drive required for the narrow grip may be greater and result in increased effort. As has been demonstrated in many previous studies, greater central drive generally leads to increases in perceived heaviness (e.g., Cafarelli \& Bigland-Ritchie, 1979; Gandevia \& McCloskey, 1977a, 1977b; Jones \& Hunter, 1983). The putative increase in efficiency in the wide grip could be due to passive spring-like properties of hand muscles and tendons. When grip width increases, the muscles tend to close the hand (flexors) and their tendons stretch, result- 

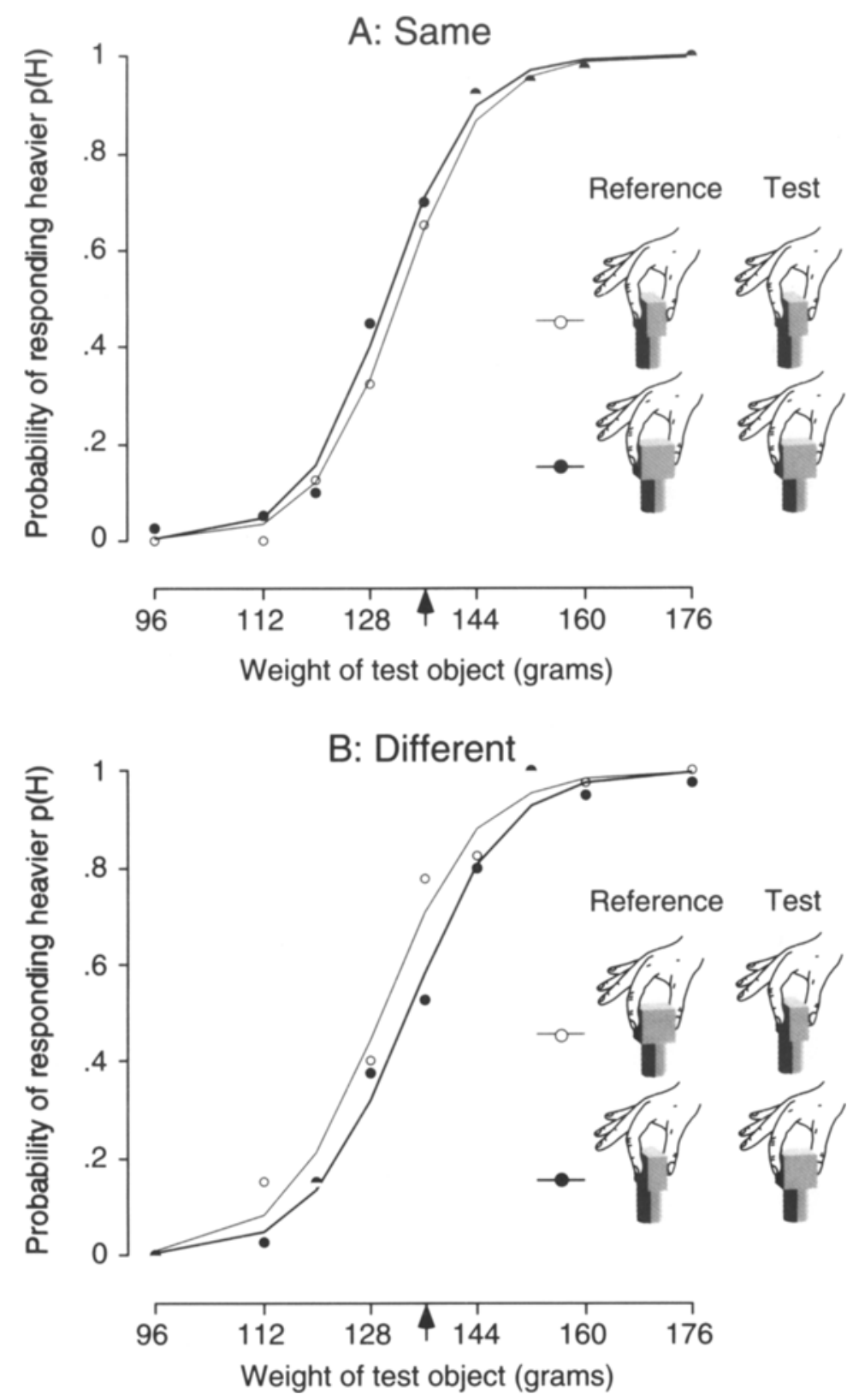

Figure 2. Group $(n=40)$ psychometric functions for weight discrimination judgments when the test and reference objects were lifted with grasps of equal width (A) or different widths $(B)$. Curves represent the probability of responding that the test object is heavier than the previously lifted reference object. When the test and reference objects are lifted with grasps of different width, the probability of responding that the test object is heavier than the reference is greater when the test object is grasped with a narrow grasp.

ing in an increase in passive elastic force and reducing the active force that needs to be generated by central motor commands.

Amazeen and colleagues (Amazeen, 1997; Amazeen \& Turvey, 1996) have shown that rotational dynamics have an important contribution to weight perception. In the present experiments, we attempted to reduce any effects of rotational dynamics, as well as torque, by in- structing the participants to lift the objects vertically and encouraging them to grasp the centers of the objects such that the grip axis would be aligned vertically with the center of mass. However, small torques (both static and dynamic) would nevertheless be observed at the fingertips (see Kinoshita, Bäckström, Flanagan, \& Johansson, 1997). Any misalignments of the grip axis may have been greater in the narrow grip simply because of the greater 
available surface area for grasping. Thus, it is possible that torques and rotational dynamics may have contributed to the increase in perceived weight observed with the narrow grip. However, we would emphasis that, given the instructions to the participants, any differences in these quantities between the narrow and wide grips would be expected to be very small.

According to the size-weight illusion, a small object lifted with a narrow grip will be judged to be heavier than a large object, of equal weight, lifted with a wide grip. People perceive the larger object to be lighter even though they expect it to be heavier prior to lifting (Ross, 1969). The size-weight illusion indicates that perceived weight depends on object size. The present results suggest that grip width per se may contribute to the difference in perceived weight between the small and large objects.

\section{EXPERIMENT 2 \\ Effect of Grip Width and Viewed Object Size on Perceived Weight}

In Experiment 1, we showed that participants judged an object grasped with a wide precision grip to be lighter than an object grasped with a narrow precision grip. We suggested that objects are perceived to be lighter when grasped with a wide grip because this grip requires less central drive or effort. However, another possibility is that the effect of grip width is influenced by the size-weight illusion even though we kept object size constant. According to this view, the object grasped with the wide grip is perceived, at some level, to be bigger despite the fact that the objects grasped with the wide and narrow grips were visibly equal in size. As a result, participants may judge the object grasped with the wide grip to be lighter because it is lighter than would be expected given its (haptically) larger size.

The aim of Experiment 2 was to distinguish between these competing hypotheses. In Experiment 1, the participants reached forward (in the sagittal plane) to grasp the objects. Thus, the wide side of the object was in view when using the narrow grip, and the narrow side of the object was in view when using the wide grip. As a result, haptic information about the "size" of the object was in conflict with visual information about the "size" of the object. If a size-weight mechanism was at work, the effects might reasonably be expected to be relatively small because of this conflict. On the other hand, if visual and haptic information were matched, then any effects associated with the size-weight illusion might be expected to be larger.

In Experiment 2, we included a condition in which the objects were placed at the participant's side such that the wide side of the object was in view when the wide grasp was employed and the narrow side of the object was in view when the narrow grasp was used. This corresponded to the matched condition (Figure 3 ). We also replicated Experiment 1 by including an unmatched condition (Figure 3 ) in which the objects were placed in front of the participants such that visual and haptic information related to object size were in conflict.

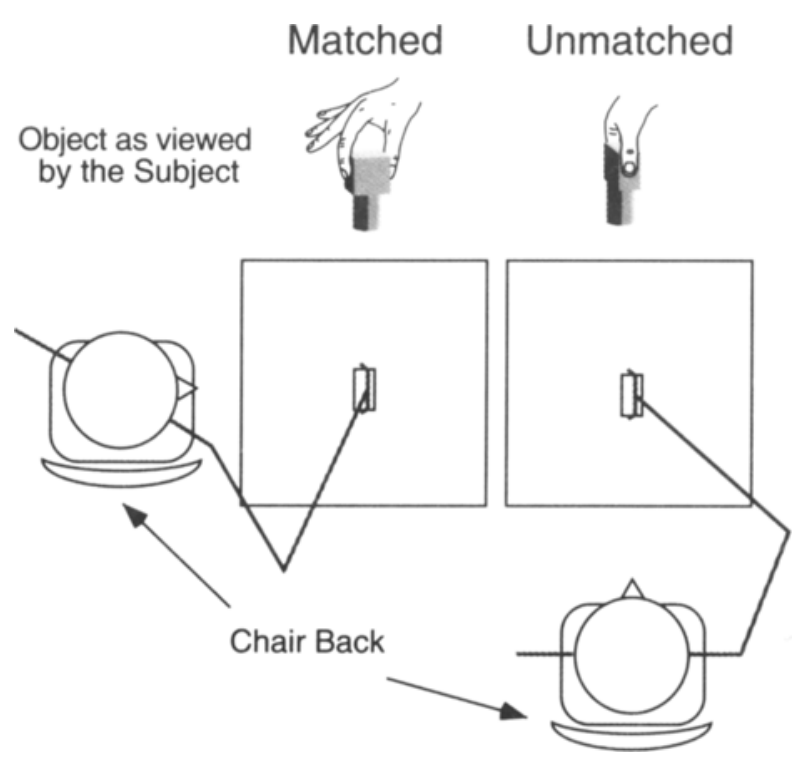

Figure 3. Schematic diagram illustrating the two experimental conditions tested in Experiment 2 . In both cases, the participant is shown grasping the object (gray rectangle) with the wide grip. (The object was rotated $90^{\circ}$ when it was grasped with a narrow grip.) In the matched condition (left), the participant viewed the wide side of the object, whereas in the unmatched condition (right), the participant viewed the narrow side of the object.

\section{Method}

Participants. Forty participants ( 29 women and $11 \mathrm{men}$ ) from the Queen's University Psychology 100 subject pool completed this experiment. These participants were not the same ones who completed Experiment 1. Informed consent was obtained prior to testing. Again, the participants received credit toward their final grade in Psychology 100 in exchange for participating. None of the participants reported sensory or motor impairments. The participants in this experiment also participated in Experiment 5.

Stimuli. The stimuli were the same as those used in Experiment 2 and consisted of rectangular blocks of wood mounted on film canisters of varying weight.

Procedure. In this experiment, we only included conditions in which the reference and test objects were grasped with different grip widths. Thus, in both matched and unmatched conditions, the participants completed two sets of nine lifts. In one set, the reference object was grasped with a narrow grip and the test objects were grasped with a wide grip (narrow-wide condition), and, in the other set, the reference was grasped with a wide grip and the test objects were grasped with a narrow grip (wide-narrow condition). The order of lift sets (wide-narrow and narrow-wide) and the order of conditions (matched or unmatched) were counterbalanced across participants. In all lift series, the order of test objects was randomized. As in Experiment 1, in each trial, the participants lifted the reference object followed by the test object.

Analysis. The logit model described in Experiment 1 was again used here. The dummy variable representing the grip width used for the test object was again coded as follows: $G=-$ ! for the narrow grip, and $G=1$ for the wide grip.

\section{Results and Discussion}

Figure 4 shows the $p \mathrm{H}$ curves obtained for the widenarrow lifts (open circles, thin lines) and the narrowwide lifts (filled circles, thick lines) in both unmatched (Figure 4A) and matched (Figure 4B) conditions. In the 


\section{A: Unmatched}

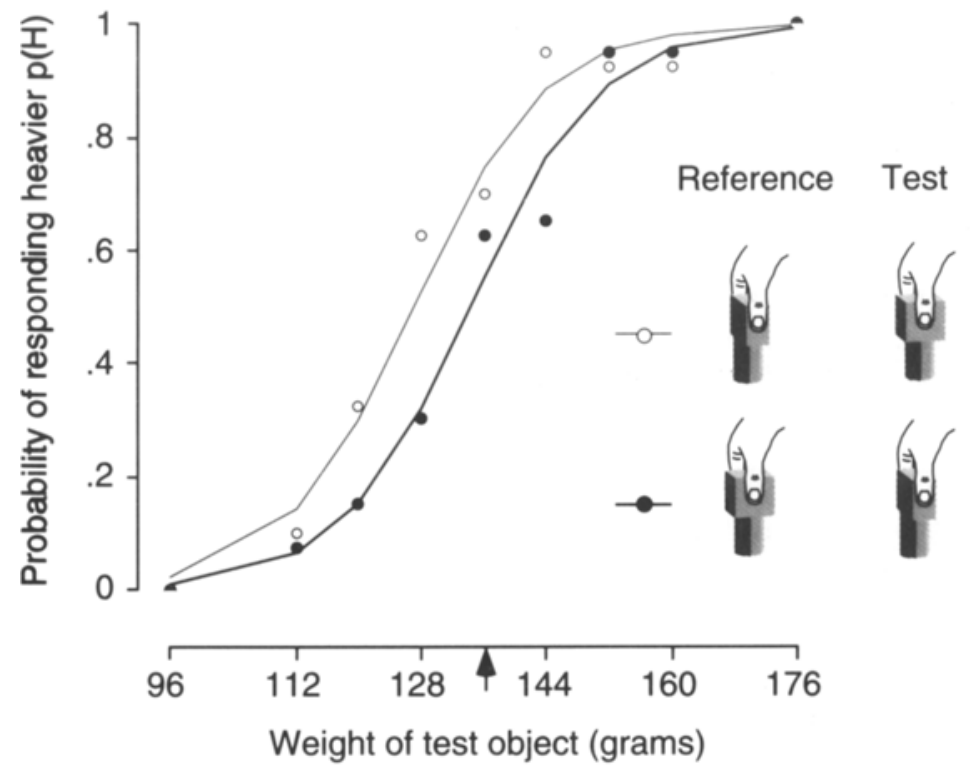

\section{B: Matched}

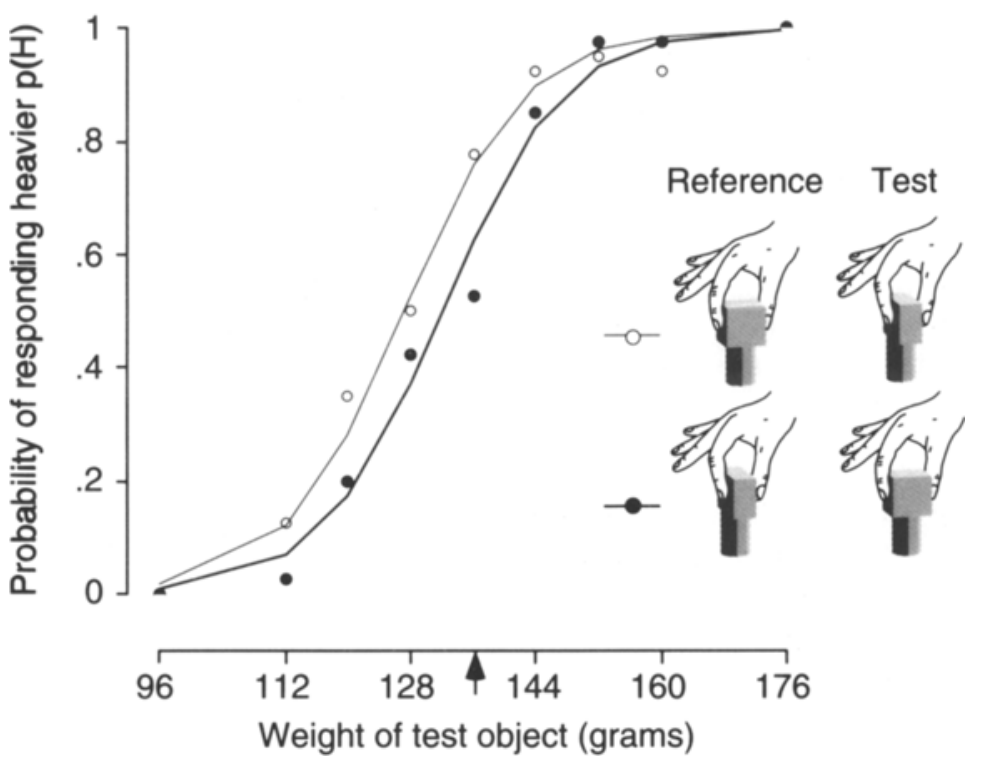

Figure 4. Group ( $n=40$ ) psychometric functions for weight discrimination judgments when the width of the grasp used to lift the test and reference objects was different. In the matched condition $(B)$, the visual width of the object matched the grasp width, whereas in the unmatched condition (A), this was not the case. Curves represent the probability of responding that the test object is heavier than the previously lifted reference object. In both the matched condition and the unmatched condition, the probability of responding that the test object is heavier than the reference is greater when the test object is grasped with a narrow grasp.

unmatched condition (corresponding to Experiment 1), we again see that objects are judged to be lighter when lifted with the wide grip than when lifted with the narrow grip. This was confirmed by the regression analysis show- ing a significant difference in the horizontal position of the two $p \mathrm{H}$ functions $[t(17)=-3.74, p<.002]$. The difference between $p \mathrm{H}$ curves was also reliable in the matched condition $[t(17)=-2.65, p=.017]$. For the 
unmatched condition, the test of parallelism did not indicate a significant interaction between the weight and grip width used for the test object $\left[\chi^{2}(1)=0.67, p=.413\right]$. However, in the matched condition, a significant interaction was observed $\left[\chi^{2}(1)=4.17, p=.041\right]$. Changes in $p \mathrm{H}$ were slightly less sensitive to changes in weight in the narrow-wide condition than in the wide-narrow condition. (Note that the estimated $p \mathrm{H}$ functions shown in Figure 4, and in all figures, are based on the additive model given in Equation 1.) Figure 4 shows that, despite the significant interaction, the additive model provides a reasonably good fit to the data.

The shifts between the $p \mathrm{H}$ functions in the unmatched and matched conditions correspond to differences in perceived weight of the test objects of 3.61 and $2.42 \mathrm{~g}$, respectively. Thus, on the average, in the unmatched and matched conditions, respectively, an object grasped with the wide grip had to be 3.61 and $2.42 \mathrm{~g}$ heavier than an object grasped with a narrow grip to be perceived to be the same weight.

Logit analysis was also used to directly compare the narrow-wide $p \mathrm{H}$ curves from the unmatched and matched conditions and the wide-narrow $p \mathrm{H}$ curves from these two conditions. The analysis did not yield a significant difference between conditions for either the narrow-wide trials $[t(17)=-1.28, p=.216]$ or the wide-narrow trials $[t(17)=0, p=1]$. (In the latter case, the two estimated $p \mathrm{H}$ functions were identical.)

The results of Experiment 2 argue against the hypothesis that grip width influences perceived weight through the haptic size-weight illusion. We reasoned that if objects lifted with a wide grip are judged to be lighter because of the haptic size-weight illusion, then we should be able to manipulate the size of the illusion by varying the visual size of the object. However, we found that visual size cues did not influence the effects of grip width on perceived weight. Of course, we cannot completely rule out the possibility that the haptic size-weight illusion was at work. However, this possibility requires that there be no conflict between the visual and haptic size-weight illusions or that the haptic illusion completely dominates the visual illusion, at least in the present task.

On the basis of the above results, we suggest that grip width can influence perceived weight because of changes in the effort required to generate the required grip force. However, we cannot rule out the possibility that grip width acts as a conditioned cue for perceived weight. According to this view, participants would expect an object to be heavier when grasped with a wide grip than when grasped with a narrow grip (based, presumably, on experience). When the weights are in fact equal, they would perceive the opposite of what they expect (see Ross, 1969). The material-weight illusion (Seashore, 1899; Wolfe, 1898) and the color-weight illusion (De Camp, 1917) provide examples, unrelated to object size, where observers perceive the opposite of what they expected before lifting the weights. (Observers expect metal or dark objects to be heavier than wooden or bright objects of equal weight and size but perceive the former to be lighter.)

\section{EXPERIMENT 3 \\ Effect of Number of Digits on Perceived Weight}

The aim of Experiment 3 was to test the prediction that an object grasped with five digits would be judged to be lighter than an object grasped with two digits. Kinoshita, Kawai, and Ikuta (1996) and Kinoshita, Murase, and Bandou (1996) have shown that when lifting objects with the digits at the sides, the total normal force decreases with the number of digits employed. On the basis of this result, we hypothesized that an object would be judged heavier when lifted with two digits than when lifted with five digits because the total normal force, and, hence, the total effort required to perform the task, would be greater. We compared a precision grip in which the object was grasped between the distal tips of the index finger and thumb with a grip in which the tips of all five digits contacted the object (Figure 1B). In both grips, the fingertips contacted the object on the side.

\section{Method}

Participants. The 40 participants were the same as those described in Experiment 1.

Stimuli. The objects consisted of plastic cylindrical Playdough containers. The height of the containers was $7.9 \mathrm{~cm}$, and the diameter was $6.1 \mathrm{~cm}$. The containers were filled with Playdough with tire weights embedded, and the interior of each container was lined with black paper to ensure that the participants would not visually detect the amount of Playdough contained. As in Experiment 1, there were nine test objects ranging in weight from 96 to $176 \mathrm{~g}$ and a reference object weighing $136 \mathrm{~g}$, the central values of the test weights.

Procedure. Each participant first completed two sets of lifts using the same grip to lift the reference and test objects (two-two and five-five) and then two more sets of lifts using different grips for the reference and test objects (two-five and five-two). The order with the same trials and the different trials was counterbalanced across participants, and the test objects were presented in random order within each lift series. The procedure for each trial was the same as described above. That is, the participants were asked to lift the reference object followed by the test object and then indicate whether the test object was lighter or heavier than the reference.

Analysis. Logit regression analysis, described above, was used to test for differences between $p \mathrm{H}$ functions obtained for different test object grips in both the same and different trials. The dummy variable representing the grip type used for the test object was coded as follows: $G=-1$ for two digits, and $G=1$ for five digits.

\section{Results and Discussion}

Figure 5A shows the $p \mathrm{H}$ values and estimated functions obtained when both the reference and the test objects were lifted with either the two-digit grip (open circles, thin lines) or the five-digit grip (filled circles, thick lines). No reliable difference was found between the two $p \mathrm{H}$ functions $[t(17)=-1.31, p=.207]$, and the test of parallelism indicated that there was not a reliable interaction between test object weight and test object grip type $\left[\chi^{2}=1.89, p=.169\right]$. Figure 5B shows the $p \mathrm{H}$ curves estimated when the reference and test objects were lifted with different grips. As can be seen in the figure, objects were judged to be lighter when lifted with a five-digit grip than when lifted with a two-digit grip, and logit analysis confirmed that the two $p \mathrm{H}$ curves are reli- 

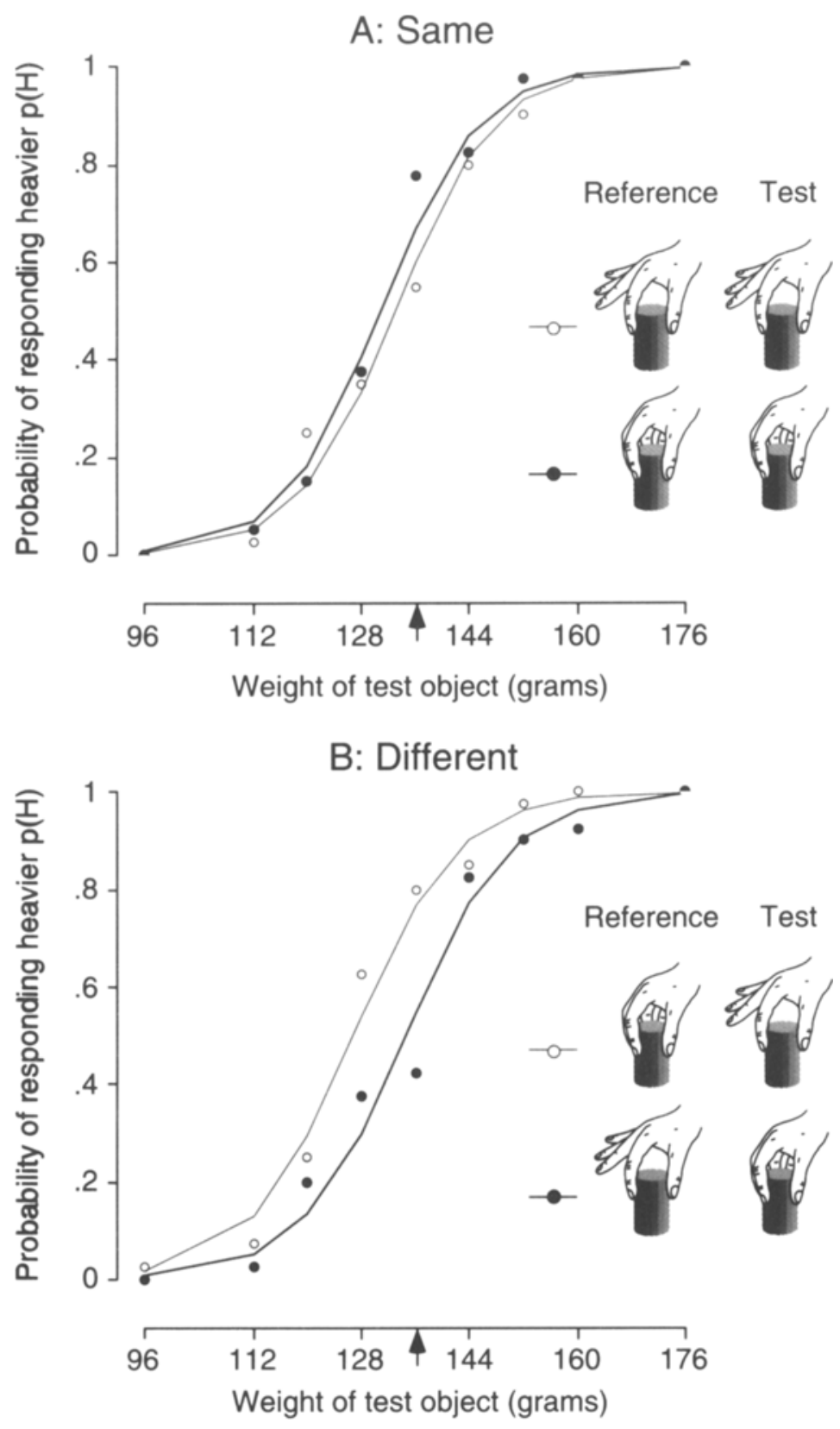

Figure 5. Group $(n=40)$ psychometric functions for weight discrimination judgments when the test and reference objects were grasped with the same number of digits (A) or with different numbers of digits (B). Curves represent the probability of responding that the test object is heavier than the previously lifted reference object. When the test and reference objects are lifted with different numbers of digits, the probability of responding that the test object is heavier than the reference is greater when the test object is grasped with two digits.

ably different $[t(17)=4.17, p<.001]$. The test of parallelism was not significant $\left(\chi^{2}=0.96, p=.327\right.$ ), indicating that there was no interaction between weight and grip type. The horizontal shift between the two $p \mathrm{H}$ functions corresponds to a difference in perceived weight of $7.55 \mathrm{~g}$, indicating that, on average, an object grasped with the five-digit grip would have to be $7.55 \mathrm{~g}$ heavier than an object grasped with the two-digit grip to be perceived to be of equal weight. By comparison, the difference in perceived weight (not significant) between the two-two and five-five conditions was $-2.23 \mathrm{~g}$.

The results of Experiment 3 clearly show that objects were perceived to be lighter when lifted with five digits than when lifted with just the index finger and thumb. We 
suggest that this may be because the total normal force used in the two-digit grasp is greater than that used in the five-digit grasp (Kinoshita, Kawai, \& Ikuta, 1996; Kinoshita, Murase, \& Bandou, 1996). We assume that the total normal force determines the effort involved in lifting and that weight perception is influenced by this effort.

A two-digit grasp is inherently less stable than a fivedigit grasp, and this may explain why normal forces are greater in the two-digit grasp. The greater need to enhance grasp stability when lifting with two digits may also result in increased cocontraction of opposing hand muscles that would stiffen the digits. Such increases in muscle cocontraction could also contribute to a greater sense of effort when lifting with two digits.

A second reason why normal forces are greater in the two-digit grasp may be related to fingertip torques. In a two-digit grasp, if the grip axis (the line joining the index finger and thumb) does not pass through the center of the object, then substantial torques may be generated at the digit contacts. The torques require increases in normal force to prevent rotational slips (Kinoshita et al., 1997). Any misalignment of the grip axis would also create rotational dynamics (about the grip axis) when lifting, and this, in addition to torque, could contribute to perceived heaviness (see Amazeen, 1997; Amazeen \& Turvey, 1996).

An alternative explanation for the results of this experiment is that there is incomplete integration of efferent and/or afferent signals across the muscles involved in lifting. That is, the central nervous system may not fully integrate either efferent commands to the different digits or afferent signals arising from the receptors associated with the digits. Thus, the total effort associated with the five-digit grip would be less. A related possibility is that the perceptual system is dominated by efferent and/or afferent signals associated with the index finger and thumb because these are the primary digits used in many manipulation tasks. In this case, an object grasped with the fivedigit grasp would be judged lighter because the forces generated by these two digits would be considerably smaller.

\section{EXPERIMENT 4 \\ Effect of Grip Surface Angle on Perceived Weight}

In Experiment 4, we examined whether the angle of the object surfaces contacted by the digits affects perceived weight. In particular, we asked participants to compare the weights of wedge-shaped objects grasped with the index finger and thumb either on the angled or vertical sides (Figure 1C). Jenmalm and Johansson (1997) have shown that the fingertip forces required to lift the object are greater when the grasp surfaces are angled than when they are vertical. Because an increase in total force presumably requires an increases in total effort (central drive), we hypothesized that the perceived weight of a given object would increase when grasping the angled sides.

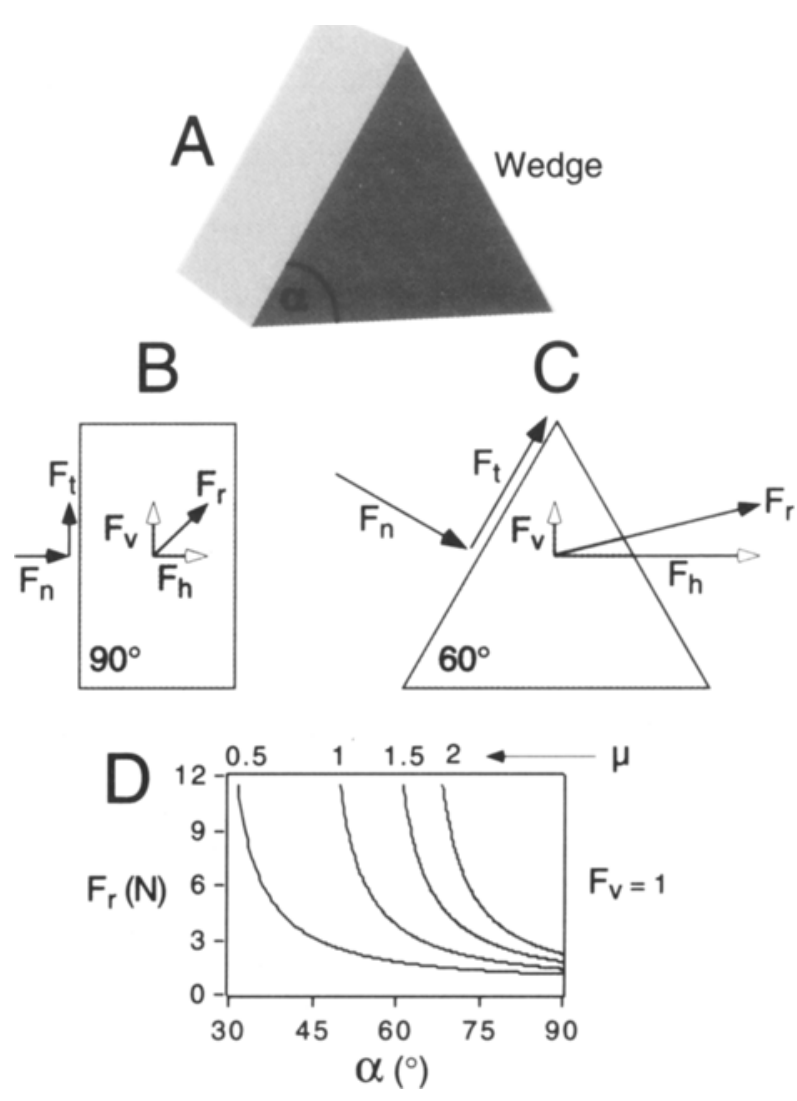

Figure 6. Forces required to support the wedge-shaped object (A) when grasping either the vertical (B) or angled (C) sides. The forces of one digit are shown. Greater normal $\left(F_{\mathrm{n}}\right)$ and tangential $\left(F_{t}\right)$ forces are required to lift when grasping the angled $\left(60^{\circ}\right)$ sides. Decomposing the force into vertical $\left(F_{v}\right)$ and horizontal $\left(F_{h}\right)$ components reveals that the latter is greater when grasping the angles, as is the magnitude of the resultant $\left(F_{r}\right)$ or total force generated by the digit. (D) The resultant force is shown as a function of angle $(\alpha)$ for different coefficients of friction $(\mu)$ between the digit and object.

The fingertip forces required to lift a wedge when grasping the angled or vertical sides are considered in Figure 6. In this experiment, the angled side of the wedge $(\alpha)$ was $60^{\circ}$ from the horizontal. The minimum forces required to lift the object when grasping the vertical and angled surfaces are illustrated in Figures $6 \mathrm{~B}$ and $6 \mathrm{C}$, respectively. (Only the forces of one digit are shown. These are assumed to be mirrored by the forces of the opposing digit.) The minimum forces are constrained by the weight of the object and the coefficient of friction $(\mu)$ between the skin and object. The vertical force $\left(F_{\mathrm{v}}\right)$ exerted by each digit is assumed to be half the weight of the object, and, for purposes of illustration, $\mu$ is assumed to be 1 . The normal force $\left(F_{\mathrm{n}}\right)$ required to prevent slip is given by $F_{\mathrm{n}}=\mu F_{\mathrm{t}}$, where $F_{\mathrm{t}}$ is the tangential force. As can be seen in the figure, in order to generate the required vertical force, $F_{\mathrm{n}}$ and $F_{\mathrm{t}}$ must be much greater when grasping the angled surface than when grasping the vertical surface. The forces can also be expressed in terms of their vertical and 
horizontal components. The horizontal force $\left(F_{\mathrm{h}}\right)$ is much larger when grasping the angled surface and the magnitude of the resultant force $\left(F_{\mathrm{r}}\right)$ vector (i.e., total force) is also greater for the angled surfaces.

The minimum resultant force $\left(F_{\mathrm{r}}\right)$ is a function of the vertical force $\left(F_{\mathrm{v}}\right)$, the coefficient of friction $(\mu)$, and the angle $(\alpha)$ of the contact surface:

$$
F_{\mathrm{r}}=\frac{\sqrt{F_{\mathrm{v}}^{2}\left(\mu^{2}+1\right)}}{\sin (\alpha)-\mu \cos (\alpha)} .
$$

The graph shown in Figure 6D shows $F_{\mathrm{r}}$ as a function of $\alpha$ for different values of $\mu$, where $F_{\mathrm{v}}$ is assumed to be $1 N$. The coefficient of friction between the skin and a flat fine-grain sandpaper surface is typically about 0.8 , and this value is quite stable across participants (e.g., Johansson \& Westling, 1984). This means that, for a given object weight, the minimum $F_{\mathrm{r}}$ will be about 2.7 times as large when grasping the angled $\left(60^{\circ}\right)$ surfaces as when grasping the vertical $\left(90^{\circ}\right)$ surfaces. Because participants employ normal forces that are only a little above the minimum value (Jenmalm \& Johansson, 1997; Johansson \& Westling, 1984), we can expect the actual fingertip forces to also be substantially greater for the angled surface than for a flat vertical surface. As noted above, this prediction has been confirmed experimentally (Jenmalm \& Johansson, 1997). On the basis of these observations, we expected the object to be judged heavier when lifted by the angled surfaces than when lifted by the vertical surfaces because of the additional force, and, hence, effort, required.

\section{Method}

Participants. The same 40 participants described in Experiment 1 took part in this experiment.

Stimuli. The stimuli were a set of wooden equilateral triangular blocks (wedges), $6.0 \mathrm{~cm}$ wide at the base, $5.2 \mathrm{~cm} \mathrm{high,} 3.5 \mathrm{~cm}$ deep, and with interior angles of $60^{\circ}$. All surfaces of the wedges were covered by fine-grain sandpaper (no. 400), which provided enough friction to prevent slipping when lifting. These wedges were mounted on 35-mm-film canisters filled with Playdough and tire weights. The weights of the reference and nine test objects were the same as those used in Experiment 1.

Procedure. The participants were required to use a precision grip (thumb and index finger) to grasp the objects either on the vertical sides or on the angled sides. The experimenter demonstrated these different grips. The participants were asked to grasp the object by contacting the middle of the contact surfaces, halfway up the object. The width of the block at half the height was $3.4 \mathrm{~cm}$, which was close to the depth of the block $(3.5 \mathrm{~cm})$. Thus, by requiring the participants to grasp halfway up the object, we could control for grip width. The experimenter monitored the participants to ensure that they grasped at the appropriate location.

Each participant completed two sets of same trials in which he/she grasped the reference and test objects on either the angled (angled-angled) or the vertical (vertical-vertical) sides followed by two sets of difference trials in which the reference was grasped on the angled sides, and the test was grasped on the vertical sides (angled-vertical), or vice versa (vertical-angled). Again, in each trial, the participant was asked to indicate whether the test object was lighter or heavier than the previously lifted reference object where both objects were lifted with the preferred hand.
Analysis. The logit model described in Experiment 1 was used to test for differences in the probability functions across grips where the dummy variable representing the grip type used for the test object was coded as follows: $G=-1$ for the angled surface, and $G=1$ for the vertical surface.

\section{Results and Discussion}

Figure 7 shows $p \mathrm{H}$ values and estimated $p \mathrm{H}$ functions obtained when the test object was grasped on either the angled sides (open circles, thin lines) or the vertical sides (filled circles, thick lines). Figure 7A shows data for same trials in which the reference and test objects were both grasped on the same sides. Figure 7B shows data for different trials in which the reference and test objects were grasped on different sides. Logit analysis failed to reveal significant differences between the $p \mathrm{H}$ curves for either the same trials $[t(17)=-1.45, p=.166]$ or the different trials $[t(17)=-1.60, p=.129]$. The test of parallelism did not reveal a reliable interaction between weight and grip type for either the same $\left[\chi^{2}(1)=0.05\right.$, $p=.822]$ or different $\left[\chi^{2}(1)=0.13, p=.719\right]$ trials.

The results clearly do not support the hypothesis that an object grasped on its angled sides is perceived to be heavier than the same object grasped on its vertical sides. Even though substantially more force is required to lift when grasping the angled sides, the additional effort does not seem to influence perceived weight. This finding contrasts with our earlier work on lifting objects with different surface textures (Flanagan et al., 1995) in which we showed that the perceived weight of an object increases with surface slipperiness and, hence, the grip force required to prevent slip. It seems that the perceptual system is able to take object shape (i.e., the angle of the contact surfaces) into account when making judgments of weight but does not take frictional differences associated with surface material into account.

The different effects of object shape and friction on weight perception may be related to the use of visual cues to generate expectations about the forces required to lift the object. Whereas visual cues about object shape are used to control fingertip forces in an anticipatory fashion (Jenmalm \& Johansson, 1997), it appears that visual cues about friction are not used for anticipatory force control (Edin, Westling, \& Johansson, 1992; Johansson $\&$ Westling, 1984). When lifting objects of varying shape, expectations about the required forces-obtained from visual cues - may enable the perceptual system to separate vertical forces (e.g., weight) from other forces that could otherwise bias weight perception. We will return to this issue in the General Discussion section.

We noticed that when grasping the angled sides, some participants tended to flatten the tips of their digits against the contact surface such that the total surface area in contact with the object appeared to be larger than when grasping the vertical sides. If weight perception is influenced by pressure per unit area on the skin, this flattening of the digits could have confounded the results. The aim of Experiment 5 was to evaluate this possibility. 

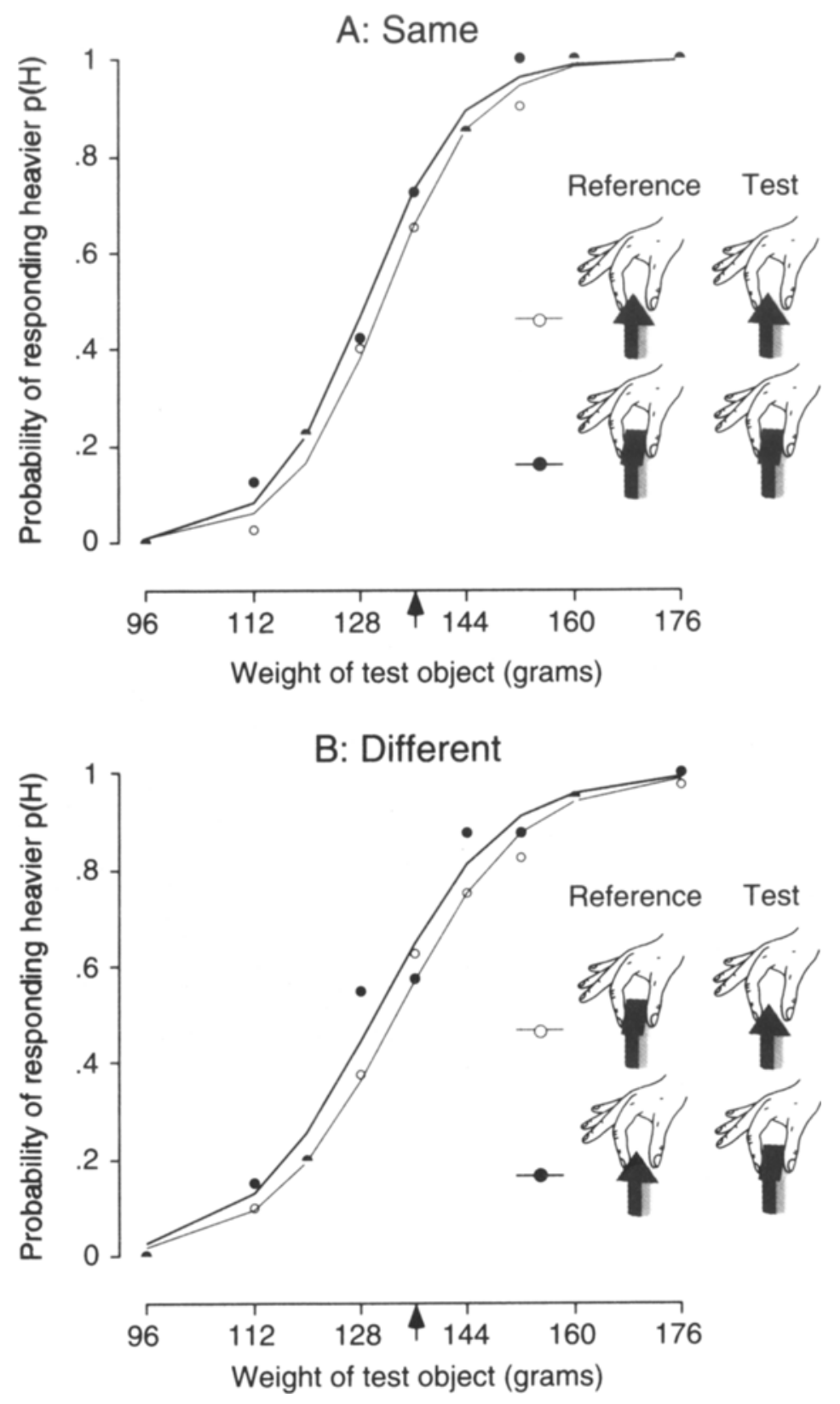

Figure 7. Group ( $n=40$ ) psychometric functions for weight discrimination judgments when the angles of the contact surfaces of the test and reference objects were the same $(A)$ or different $(B)$. Curves represent the probability of responding that the test object is heavier than the previously lifted reference object.

\section{EXPERIMENT 5}

\section{Effect of Grip Surface Area on Perceived Weight}

In Experiment 4, we had hypothesized that a wedgeshaped object would be judged to be heavier when grasped on its angled sides than when grasped on its vertical sides. However, the results failed to support this hypothesis. In watching participants lift the triangular objects, we observed that the size of the contact area between the digits and the object tended to be larger when grasping the angled sides of the object than when grasping the vertical sides of the object. This raises the question whether contact area per se might influence weight perception. The objective of Experiment 5 was to evaluate this hypothesis. Participants were explicitly instructed to grasp the angled sides of the triangular objects either with a flattened grip with a relatively large contact area or with a pinch grip with a relatively small contact area (see Fig- 
ure 1D). We hypothesized that the object would be perceived to be lighter when grasped with the large contact area than when grasped with a small contact area.

Charpentier (1891; Murray et al., 1999) investigated the effect of contact area on perceived weight by mounting an object on a series of flat lightweight support surfaces and placing the surfaces on the hand. (Thus, the total weight of the object and support was approximately constant, while the contact area varied.) Charpentier reported that the perceived weight of the object decreased with the size of the surface. However, it should be noted that there are important differences between Charpentier's experiment and the present experiment. In the former, contact area may have contributed to a haptic size-weight illusion or acted as a conditioned cue. Thus, for example, participants may have expected an object mounted on a larger contact area to be heavier. In contrast, it seems less likely that the size of the digit contact areas would have the same effect. That is, there is no obvious relation that we can think of why digit contact area would be correlated with object size or weight.

\section{Method}

Participants. The same 40 participants described in Experiment 2 took part in this experiment.

Stimuli. The stimuli were the same as those used in Experiment 4

Procedure. The participants were required to use the thumb and index finger to grasp the objects either with a flattened grip with a large contact area or with a pinch grip with a small contact area. The experimenter demonstrated these different grips. In this experiment, we only examined trials in which the reference object and the test object were grasped with different grips. Although this repre- sents a change in the experimental conditions from Experiment 4, we can think of no reason why this would confound any effect of grasp surface area on perceived weight. Each participant completed one set of trials in which the reference was grasped with a large contact area and the test was grasped with a small contact area and completed another set of trials in which the two grips were reversed. The order of sets was counterbalanced across participants, and the order of test weights within each set was randomized.

Analysis. The logit model described in Experiment 1 was used to test for differences in the $p \mathrm{H}$ functions across grips where the dummy variable representing the grip used for the test object was coded as follows: $G=-1$ for the small contact area, and $G=1$ for the large contact area.

\section{Results and Discussion}

Figure 8 shows the $p \mathrm{H}$ values and estimated $p \mathrm{H}$ functions obtained when the test object was grasped with either the large contact area (open circles, thin line) or the small contact area (filled circles, thick line). At all test weights, the $p \mathrm{H}$ was smaller when the test object was lifted with the large contact area, and logit analysis confirmed that there was a reliable difference between these curves in terms of their horizontal position $[t(17)=7.20, p<$ $.001]$. The test of parallelism did not yield a significant interaction between weight and contact area $\left[\chi^{2}(1)=\right.$ $0.47, p=.492]$. The shift between the two $p \mathrm{H}$ functions corresponds to a difference in perceived weight of $16.89 \mathrm{~g}$, indicating that an object grasped with a large contact area had to be $16.89 \mathrm{~g}$ heavier, on average, than an object grasped with a small contact area to be judged equal in weight.

The results of Experiment 5 suggest that the size of the contact area between the digits and the object influ-



Figure 8. Group ( $n=40)$ psychometric functions for weight discrimination judgments when the size of the contact area between the digits and the object was different for the test and reference objects. Curves represent the probability of responding that the test object is heavier than the previously lifted reference object. The probability of responding that the test object is heavier than the reference is greater when the test object is grasped with small contact areas. 
ences weight perception. A possible explanation for this finding is that perceived weight is influenced by sensory information that depends on pressure for unit area. Assuming that similar forces were generated with the two grips, the pressure per unit area would be greater when using the pinch grip with a relatively small contact area, and, thus, perceived weight would be greater. Alternatively, it may be that the friction between the digit and the contact surface increases with surface area, which would result in lower forces in the flattened grip and less effort (cf. Kinoshita et al., 1997).

If contact area really does influence perceived weight, then the results of Experiment 4 must be interpreted with caution. There may have been an effect of object shape (i.e., angle of the contact surfaces) on perceived weight that was countered by an effect of contact area. However, there are other possible explanations for the results of the present experiment that do not involve contact area. In particular, the pinch grip may be less stable than the flattened grip and may require more cocontraction of hand muscles, leading to a greater sense of effort.

\section{GENERAL DISCUSSION}

The hand's dual functions of manipulation and exploration are intimately linked. On the one hand, perceptual information about various object properties is required to guide skilled manipulation. On the other hand, the way in which we manipulate objects depends on the perceptual information we wish to extract (Lederman \& Klatzky, 1987). In the present study, we asked whether the way in which an object is grasped could alter the perception of a single property, the object's weight. The results clearly demonstrate that perceived weight is influenced by grasp configuration. The width or span of the grasp, the number of digits employed, and the size of the contact area between the digits and object were all shown to influence perceived heaviness. In contrast, judgments of weight were not altered by the angle of the grasp surfaces. Although we have shown that changes in grasp configuration can influence perceived weight, earlier work on "weight constancy" has stressed the opposite tendency-the fact that apparent weight changes less than might be expected from changes in the manner in lifting (Ross, 1969). Thus, the influence of grasp configuration appears to be mediated or attenuated by central perceptual mechanisms.

A central issue in research on force and weight perception concerns the relative contributions of central (efferent) and peripheral (afferent) signals. There is strong evidence that both of these signals can influence perceived force or weight, at least under certain conditions. An important efferent contribution is suggested by demonstrations that perceived weight increases when the central motor drive or "effort" required to support a given load is increased by fatigue (Jones \& Hunter, 1983), partial curarization (Gandevia \& McCloskey, 1977a), changes in muscle length (Cafarelli \& Bigland-Ritchie, 1979), or neurological disorders resulting in muscular weakness (Gandevia \& McCloskey, 1977b; Holmes, 1917). However, other studies have provided evidence for an afferent contribution. For example, Brodie and Ross (1984) reported that weight discrimination in reflex lifting (induced by tendon vibration) was significantly better than when the object was passively supported by the hand and was nearly as good as in active lifting. Because reflex-mediated lifting is assumed not to involve voluntary central motor drive, Brodie and Ross concluded that sensory receptors sensitive to muscular force (e.g., tendon organs) must contribute to weight perception. Lansing and Banzett (1993) asked subjects to attempt to generate maximal contractions of inspiratory and hand muscles while paralyzed with a neuromuscular block. When questioned after recovery, none of the subjects reported sensations of exerted force, great effort, or heaviness, which would have been expected if motor commands alone were the source of these sensations.

For the most part, the results described in this paper are broadly consistent with the view that forces only indirectly involved in lifting or supporting the object can contribute to weight perception by increasing the total force or effort required. Thus, we suggested that (1) an object is judged to be lighter when grasped with a wide grip than when grasped with a narrow grip because less effort is required to generate grip force in the wide grip, (2) an object grasped with five digits is judged to be lighter than when it is grasped with two digits because less effort is required to stabilize the digits when all five are employed, and (3) an object is perceived to be lighter when grasped with a flattened grip than when grasped with a pinch grip, again because the flattened grip is more stable. However, we also discussed alternative explanations for each of these experimental results, and we cannot rule out the possibility that factors unrelated to the sense of effort associated with grasping may have contributed to perceived weight.

We were surprised that the angle of the contact surfaces did not influence weight perception given the large increase in horizontal force required to lift using the angled sides in comparison with the vertical sides (Jenmalm \& Johansson, 1997). This result stands in marked contrast to our previous finding that objects covered in slippery material, requiring larger grip (horizontal) forces, are judged to be heavier than objects covered in less slippery material, requiring smaller grip forces (Flanagan \& Wing, 1997; Flanagan et al., 1995). This difference between angle and friction effects on perceived weight may be due to the role of visual cues and stored knowledge about object properties. It is well established that visual cues about object shape (Jenmalm \& Johansson, 1997; Wing \& Lederman, 1998) and visual and haptic cues about object size (Gordon, Forssberg, Johansson, \& Westling, 1991 a, 1991b) can be used for anticipatory fingertip force control. That is, given cues about object shape and size (from which weight can be estimated), participants begin to appropriately scale their fingertip forces right from the very start of the lift (before sensory feedback can be used to update these forces) even if they have not previously lifted 
the object. This indicates that visual information about object shape and weight is used to access stored knowledge about the force requirements for lifting. It may be that this knowledge about the relation between object properties (shape and size) and required forces is available to the perceptual system and enables the system to take these properties into account when judging weight.

Such stored knowledge may not be available to deal with object frictional properties. In contrast to object shape and size, people do not appear to use visual cues about friction to make anticipatory force adjustments when manipulating objects (Edin et al., 1992; Johansson \& Westling, 1984). This may be caused by the fact that such cues are simply unreliable. In any event, given that visual friction cues are not used, there is no reason to store knowledge about the relation between friction and required forces. Instead, the motor system appears to rely on sensory feedback and short-term sensorimotor memory to scale fingertip forces appropriately for different frictional conditions (Johansson \& Westling, 1984). The absence of such stored knowledge may explain why the increased effort required to lift a slippery object results in an increase in perceived weight.

The finding that perceived weight is influenced by grip width and the number of digits used in grasping suggests that the perceptual system is not able to adjust for these biomechanical factors. In the case of grip width, it appears that participants do not take account of change in muscle efficiency with changes in muscle length, a finding consistent with previous studies in which muscle length has been manipulated (Cafarelli \& Bigland-Ritchie, 1979). Interestingly, there is some evidence that the motor system does not always take biomechanical factors into account when controlling action. Werremeyer and Cole (1997) have reported that, during wrist flexion and extension movements with an object held in a precision grip, there are increases in grip force associated with activation of extrinsic hand muscles that contribute to both wrist rotation and grip force. These grip force increases were not necessary to prevent slip and thus may be viewed as errors that are not fully accounted for by the motor system.

In summary, the results generally support the idea that forces only indirectly involved in lifting may influence weight perception. However, this is not always the case. Under some conditions, participants are able to take these indirectly involved forces into account. Although we have offered some tentative suggestions, the question as to why some factors (e.g., surface angle) but not others (e.g., surface friction) can be taken in account remains open.

\section{REFERENCES}

Amazeen, E. L. (1997). The effects of volume on perceived heaviness by dynamic touch: With and without vision. Ecological Psychology, 9, 245-263

Amazeen, E. L., \& Turvey, M. T. (1996). Weight perception and the haptic size-weight illusion are functions of the inertia tensor. Journal of Experimental Psychology: Human Perception \& Performance, 22, 213-232.
Brodie, E. E., \& Ross H. E. (1984). Sensorimotor mechanisms in weight discrimination. Perception \& Psychophysics, 36, 477-481.

Cafarelli, E. W., \& Bigland-Ritchie, B. (1979). Sensation of static force in muscles of different length. Experimental Neurology, 65, $511-525$.

Charpentier, A. (1891). Analyse expérimentale des quelques éléments de la sensation de poids [Experimental study of some aspects of weight perception]. Archives de Physiologie Normales et Pathologiques, 3, $122-135$.

DE CAMP, J. E. (1917). The influence of color on apparent weight: A preliminary study. Journal of Experimental Psychology, 62, 347-370.

Edin, B. B., \& JoHansson, N. (1995). Skin strain patterns provide kinaesthetic information to the human central nervous system. Journal of Physiology, 287, 243-251.

Edin, B. B., Westling, G., \& Johansson, R. S. (1992). Independent control of human finger-tip forces at individual digits during precision lifting. Journal of Physiology, 450, 547-564.

Ellis, R. R., \& Lederman, S. J. (1993). The role of haptic versus visual volume cues in the size-weight illusion. Perception \& Psychophysics, 53, 315-324.

FlanaGan, J. R., \& Wing, A. M. (1993). Modulation of grip force with load force during point-to-point arm movements. Experimental Brain Research, 95, 131-143.

Flanagan, J. R., \& WING, A. M. (1995). The stability of precision grip force during cyclic arm movements with a hand-held load. Experimental Brain Research, 105, 455-464

Flanagan, J. R., \& Wing, A. M. (1997). Effects of surface texture and grip force on the discrimination of hand-held loads. Perception \& Psychophysics, 59, 111-118.

Flanagan, J. R., Wing, A. M., Allison, S., \& Spencely, A. (1995). Effects of surface texture on weight perception when lifting objects with a precision grip. Perception \& Psychophysics, 57, 282-290.

FlouRnOY, T. (1894). De l'influence de la perception visuelle des corps sur leur poids apparent [On the influence of visual perception on the apparent weight of objects]. L'Année Psychologique, 1, 198-208.

GandeviA, S. C., \& McCloskey, D. I. (1977a). Changes in motor commands as shown by changes in perceived heaviness, during partial curarization and peripheral anasthesia in man. Journal of Physiology, 272, 673-689.

Gandevia, S. C., \& McCloskey, D. I. (1977b). Sensations of heaviness. Brain, 100, 345-354.

Gordon, A. M., Forssberg, H., Johansson, R. S., \& Westling, G. (1991a). Visual size cues in the programming of manipulative forces during precision grip. Experimental Brain Research, 83, 477-482.

Gordon, A. M., Forssberg, H., Johansson, R. S., \& Westling, G. (1991b). The integration of haptically acquired size information in the programming of precision grip. Experimental Brain Research, 83, 483-488.

Hellström, A. (1985). The time-order error and its relatives: Mirrors of cognitive processes in comparing. Psychological Bulletin, 97, 35-61.

HoLmEs, G. (1917). The symptoms of acute cerebellar injuries due to gunshot injuries. Brain, 40, 461-535.

Jenmalm, P., \& Johansson, R. S. (1997). Visual and somatosensory information about object shape control manipulative fingertip forces. Journal of Neuroscience, 17, 4486-4499.

JOHANSSON, R. S. (1996). Sensory control of dextrous manipulation in humans. In A. M. Wing, P. Haggard, \& J. R. Flanagan (Eds.), Hand and brain: Neurophysiology and psychology of hand movement (pp. 381-414). San Diego: Academic Press.

Johansson, R. S., \& ColE, K. J. (1994). Grasp stability during manipulative actions. Canadian Journal of Physiolology \& Pharmacology, 72, 511-524.

JOHANSSON, R. S., \& WESTLING, G. (1984). Roles of glabrous skin receptors and sensorimotor memory in automatic control of precision grip when lifting rougher or more slippery objects. Experimental Brain Research, 56, 550-564.

JoHANSSON, R. S., \& WESTLING, G. (1987). Signals in tactile afferents from the fingers eliciting adaptive motor responses during precision grip. Experimental Brain Research, 66, 141-154.

JONES, L. A. (1986). Perception of force and weight: Theory and research. Psychological Bulletin, 100, 29-42. 
Jones, L. A., \& Hunter, I. W. (1983). Effect of fatigue on muscle sensation. Experimental Neurology, 81, 640-650.

Kilbreath, S. L., \& Gandevia, S. C. (1991). Independent digit control: Failure to partition perceived heaviness of weights lifted by digits of the human hand. Journal of Physiology, 442, 585-599.

Kinoshita, H., Bäckström, L., Flanagan, J. R., \& Johansson, R. S. (1997). Planar torque effects on grip force during precision grip. Journal of Neurophysiology, 78, 1619-1630.

Kinoshita, H., KaWai, S., \& IkUTA, K. (1996). Contributions and coordination of individual fingers in multiple finger prehension. Ergonomics, 39, 243-256.

Kinoshita, H., Murase, T., \& Bandou, T. (1996). Grip posture and forces during holding cylindrical objects with circular grips. Ergonomics, 39, 1163-1176.

LANSING, R. W., \& BANzeTt, R. B. (1993). What do fully paralyzed awake humans feel when they attempt to move? Journal of Motor Behavior, 25, 309-313.

LEDERMAN S. J., \& KLATZKY, R. L. (1987). Hand movements: A window into haptic object recognition. Cognitive Psychology, 19, 342-368.

LEMON, R. N. (1993). Cortical control of the primate hand. Experimental Physiology, 78, 263-301.

MatThews, P. B. C. (1982). Where does Sherrington's muscular sense originate? Muscles, joints, corollary discharges? Annual Review of Neuroscience, 5, 189-218.

McCullagh, P., \& Nelder, J. A. (1989). Generalized linear models (2nd ed.). London: Chapman \& Hall.

Moberg, F. (1962). Criticism and study of methods for examining sensitivity in the hand. Neurology, 12, 8-19.

Murray, D. J., Ellis, R. R., Bandomir, C. A., \& Ross, H. E. (1999). Charpentier (1891) on the size-weight illusion. Perception \& Psychophysics, 61, 1681-1685.
Rinkenauer, G., Mattes, S., \& Ulrich, R. (1999). The surfaceweight illusion: On the contribution of grip force to perceived heaviness. Perception \& Psychophysics, 61, 23-30.

Ross, H. E. (1969). When is a weight not illusory? Quarterly Journal of Experimental Psychology, 21, 346-355.

Rothwell, J. C., Traub, M. M., Day, B. L., Obeso, J. A., Thomas, P. K., \& MARSDEN, C. D. $(1982)$. Manual motor performance in a deafferented man. Brain, 105, 515-542.

SEASHORE, C. E. (1899). Some psychological statistics: 2. The material weight illusion. University of lowa Studies in Psychology, 2, 36-46.

VAN DOREN, C. L. (1998). Grasp stiffness as a function of grasp force and finger span. Motor Control, 2, 352-378.

WEBER, E. H. (1996). E. H. Weber on the tactile senses (2nd ed.; H. E. Ross \& D. J. Murray, Eds. and Trans.). London: Taylor \& Francis. (Original work published 1834)

Werremeyer, M. M., \& Cole, K. J. (1997). Wrist action affects precision grip force. Journal of Neurophysiology, 78, 271-280.

WING, A. M. (1996) Anticipatory control of grip force in rapid arm movements. In A. M. Wing, P. Haggard, \& J. R. Flanagan (Eds.), Hand and brain: Neurophysiology and psychology of hand movement (pp. 301-324). San Diego: Academic Press.

Wing, A. M., \& LEDERMAN, S. J. (1998). Anticipating load torques produced by voluntary movements. Journal of Experimental Psychology: Human Perception \& Performance, 24, 1571-1581.

WOLFE, H. K. (1898). Some effects of

size on judgements of weight. Psychological Review, 5, 25-54.

(Manuscript received August 10, 1999; accepted for publication November 9, 1999.) 